\title{
Appearance of a novel measles G3 strain in multiple European countries within a two month period, 2010
}

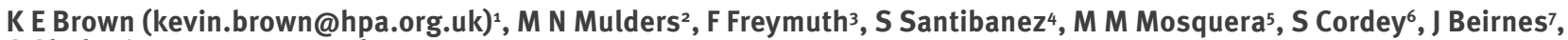
S Shulga ${ }^{8}$, R Myers ${ }^{1}$, D Featherstone ${ }^{9}$

1. Virus Reference Department, World Health Organization Global Specialised Laboratory for Measles and Rubella, Health Protection Agency - Colindale, London, United Kingdom

2. World Health Organization Regional Office for Europe, Copenhagen, Denmark

3. Centre National de Référence de la Rougeole et des Paramyxoviridae Respiratoires (National Reference Centre for measles and respiratory Paramyxoviridae), Laboratoire de virologie humaine et moléculaire (Laboratory of human and molecular virology), Centre Hospitalier Universitaire de Caen, France

4. National Reference Centre Measles, Mumps, Rubella, Regional Reference Laboratory WHO EURO, Robert Koch Institute, Berlin, Germany

5. Centro Nacional de Microbiología (National Microbiology Centre), Instituto de Salud Carlos III, Madrid, Spain

6. Swiss National Reference Centre for Emerging Viral Diseases, Division of Infectious Diseases, University of Geneva Hospitals, Geneva, Switzerland

7. Viral Exanthemata National Microbiology Laboratory, Winnipeg, Canada

8. World Health Organization Regional Reference Laboratory, European Region, Moscow, Russian Federation

9. Expanded Programme on Immunization, Department of Immunization, Vaccines and Biologicals, World Health Organization, Geneva, Switzerland

Citation style for this article:

Brown KE, Mulders MN, Freymuth F, Santibanez S, Mosquera MM, Cordey S, Beirnes J, Shulga S, Myers R, Featherstone D. Appearance of a novel measles G3 strain in multiple European countries within a two month period, 2010.

Euro Surveill. 2011;16(17):pii=19852. Available online: $\mathrm{http}: / /$ www.eurosurveillance.org/ViewArticle.aspx?Articleld=19852

This article has been published on 28 April 2011

During late 2010, a previously unrecognised strain of measles genotype $\mathrm{G}_{3}$ virus was identified in five different European countries by the World Health Organization Measles and Rubella Laboratory Network. Apart from one, none had a travel history to south-east Asia, the usual source of $\mathrm{G}_{3}$ viruses, although epidemiological links could be established between some of the cases. This case series illustrates the value of genotyping and sequencing in tracking measles infections, and identifying otherwise unrecognised chains of transmission.

\section{Measles and Rubella Laboratory \\ Network (LabNet)}

As Europe adopts its resolution to eliminate measles by 2015 [1] confirmation of clinically diagnosed measles, and characterisation of circulating viruses becomes increasingly pertinent. The World Health Organization (WHO) Measles and Rubella Laboratory Network (LabNet), was established in 2002, with the primary purpose of providing laboratory confirmation of suspected cases of measles and rubella using standardised ELISA-based IgM detection in serum or oral fluid. As of July 2010, the LabNet consists of 690 laboratories in 183 countries, all of which follow a standardised set of testing protocols and reporting procedures with a strong focus on quality assurance. The LabNet is structured in four tiers of laboratories: sub national $(n=507)$, national level $(n=161)$, regional reference $(n=19)$, and global specialised $(n=3)$.

The LabNet also supports genetic characterisation of currently circulating strains of measles viruses and is responsible for standardisation of the nomenclature and laboratory procedures that are used for genetic characterisation of wild-type measles and rubella viruses [2-4].

This agreement of a standard nomenclature and classification for describing measles virus has been instrumental in allowing comparison of viruses in different countries, and also for documenting spread within countries, monitoring viral transmission pathways and in providing evidence of progress towards measles elimination [5].

For molecular epidemiologic purposes, the WHO currently recognises eight clades (designated $A$ to $H$ ), and within these clades, there are 23 recognised genotypes, designated $A, B_{1}, B_{2}, B_{3}, C_{1}, C_{2}, D_{1}, D_{2}, D_{3}$, $\mathrm{D}_{4}, \mathrm{D}_{5}, \mathrm{D} 6, \mathrm{D}_{7}, \mathrm{D} 8, \mathrm{D} 9, \mathrm{D} 10, \mathrm{E}, \mathrm{F}, \mathrm{G}_{1}, \mathrm{G}_{2}, \mathrm{G}_{3}, \mathrm{H}_{1}$, and $\mathrm{H} 2$. There is considerable genetic variability within some genotypes (e.g. $\mathrm{B}_{3}$ and $\mathrm{H}_{1}$ ) and related viruses are referred to as clusters. The WHO recommends that the 450 nucleotides coding for the carboxyterminal 150 amino acids of the nucleoprotein $(\mathrm{N}-450)$ are the minimum amount of sequence data required for assigning a measles genotype [2-4]. A WHO Measles nucleotide sequence database MeaNS ([6], www.who-measles. org) was set up as a repository for sequence information, with tools to allow genotyping and phylogenetic analysis of measles viruses sequences found globally.

Genotyping and sequencing of measles viruses is recommended by the WHO for at least $80 \%$ of all laboratory-confirmed outbreaks [7] and in countries where measles is no longer endemic, is encouraged for all 
sporadic cases. Molecular characterisation of such cases provides the information necessary to determine whether they are part of a single cluster or due to multiple importations, and to identify sources of infection. However, unless appropriate samples are collected (generally throat swab, urine sample, or increasingly common in Europe, an oral fluid sample) genotype information is not always available. In the past three years, six different genotypes of measles virus have been identified in Europe (B3, D4, D5, D8, D9, H1) with large outbreaks associated with $\mathrm{B}_{3}, \mathrm{D}_{4}$ and $\mathrm{D}_{5}$ in many countries [8].

\section{Recent infections with measles virus G3 in Europe}

Measles genotype $\mathrm{G}_{3}$ is generally associated with measles infections in south-east Asia, or in sporadic cases with links to south-east Asia [4]. There had been no reported cases of measles $\mathrm{G}_{3}$ in Europe since 2006. However, in the last four months of 2010 there have been a total of 25 sporadic (i.e. epidemiologically unlinked) or epidemiologically linked cases, all with viruses with identical sequence belonging to genotype $\mathrm{G}_{3}$, in several different countries in Europe.

The first case, Patient A was a non-vaccinated German adult who was on a roundtrip from Germany to southeast Asia, during the first half of September 2010, and returned to Germany via London on 17 September 2010. Patient $A$ remembered that a passenger sitting one row in front of them had influenza-like symptoms and was coughing frequently, but otherwise had no known contact with potential infectious measles virus carriers. Ten days later Patient A felt ill, developed a rash after two more days and was hospitalised. Measles was confirmed by detection of measles virus RNA in oral fluid and urine sample [9].

During the same period of symptom onset for Patient A, cases of measles were also detected in Spain, England and France (Figure 1).

The Spanish case (Patient B) returned to Spain on 20 September 2010 after having travelled to London. Like Patient A, Patient B developed the prodrome on 27 September, with rash onset on 3 October. These dates indicate a potential infection of both cases in London around 17 September.

The third case was a college student in London (Patient C) with no history of travel outside the United Kingdom (UK), who became unwell on 28 September and who had rash onset on 2 October. Patient $C$ subsequently infected two students at the same college in London (Patient D, onset date 12 October, and Patient E, onset date 14 October). Patient D had travelled to Switzerland and became unwell and was diagnosed with measles there. Subsequently there were two further cases of measles $G_{3}$ virus infection, a household contact of Patient D (Patient F, onset date 27 October) and another case (Patient G, onset date 29 October) with no identified links, but living in the same town.

\section{FIGURE 1}

Measles infections with known epidemiological links in different countries, 2010

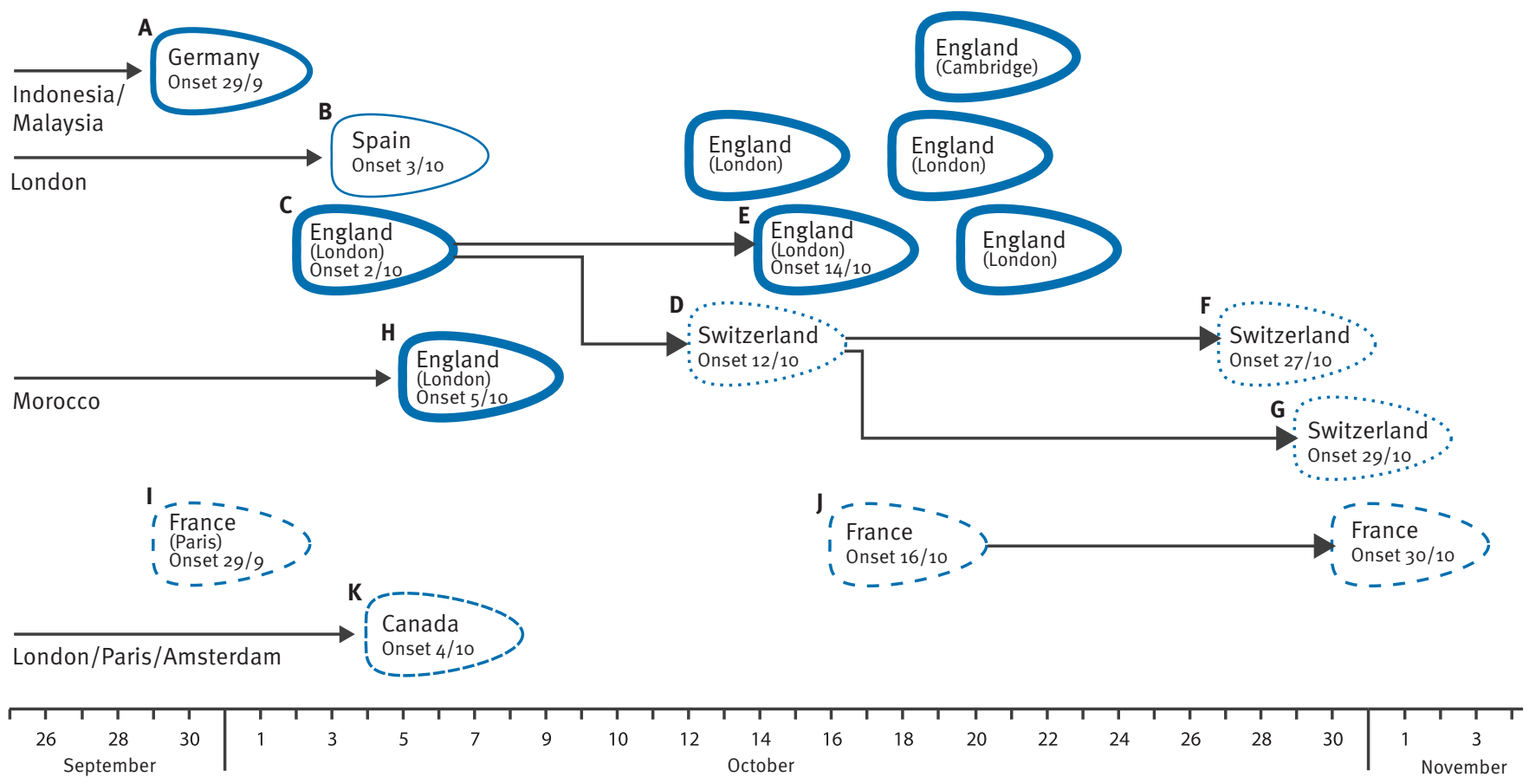

2010

The different styles of the outlines indicate the different countries where the cases were at the time of infection. 
A fourth patient in the UK (Patient $\mathrm{H}$ ) also developed measles with a $\mathrm{G}_{3}$ genotype at the beginning of October 2010. Patient $\mathrm{H}$ had spent holidays in North Africa and returned to the UK on 20 September. The prodrome began on 1 October with rash onset four days later, suggesting infection at the time of travel or shortly after, and although this case did not live in London, they had travelled through London on the way home. Subsequently, over the next two months there were four further sporadic $\mathrm{G}_{3}$ measles cases, all with no history of travel outside south-east England. There were no further cases in the UK for the rest of 2010.

The French patient (Patient I) was a one year-old nonvaccinated infant who lived in the area of Paris, and developed rash on 29 September. Like Patient C, there was no history of recent travel or known epidemiological link to anyone with measles. On 16 October there was a second $\mathrm{G}_{3}$ measles confirmed case in France (Patient J), although no links between the two cases could be determined. Subsequently, there were a further nine sporadic cases of $\mathrm{G}_{3}$ measles identified in France up to the end of 2010, but given the widespread measles activity in France [10] links between these cases have been difficult to identify.

Lastly, a visitor from Canada with a history of one dose of measles-containing vaccine as an infant, (Patient $\mathrm{K}$ ) travelled to London on 10 September and then spent holidays in London, Paris and Amsterdam. Patient $\mathrm{K}$ also developed measles with a $\mathrm{G}_{3}$ genotype, with onset of rash on 4 October, suggesting infection around 20 September before having returned to Canada.

\section{Sequence analysis}

Genotyping and sequencing was performed, by the respective national laboratory on a variety of samples from these patients. Sequencing of the recommended minimum 450 nucleotides [3] was performed and confirmed that they were all $\mathrm{G}_{3}$ sequences. Comparison of the sequences confirmed that all the samples, including the one from the German patient with travel links

\section{FIGURE 2}

Phylogenetic tree of representative sequences from the recent G3 measles cases, compared with other historic G3 measles cases, 2010

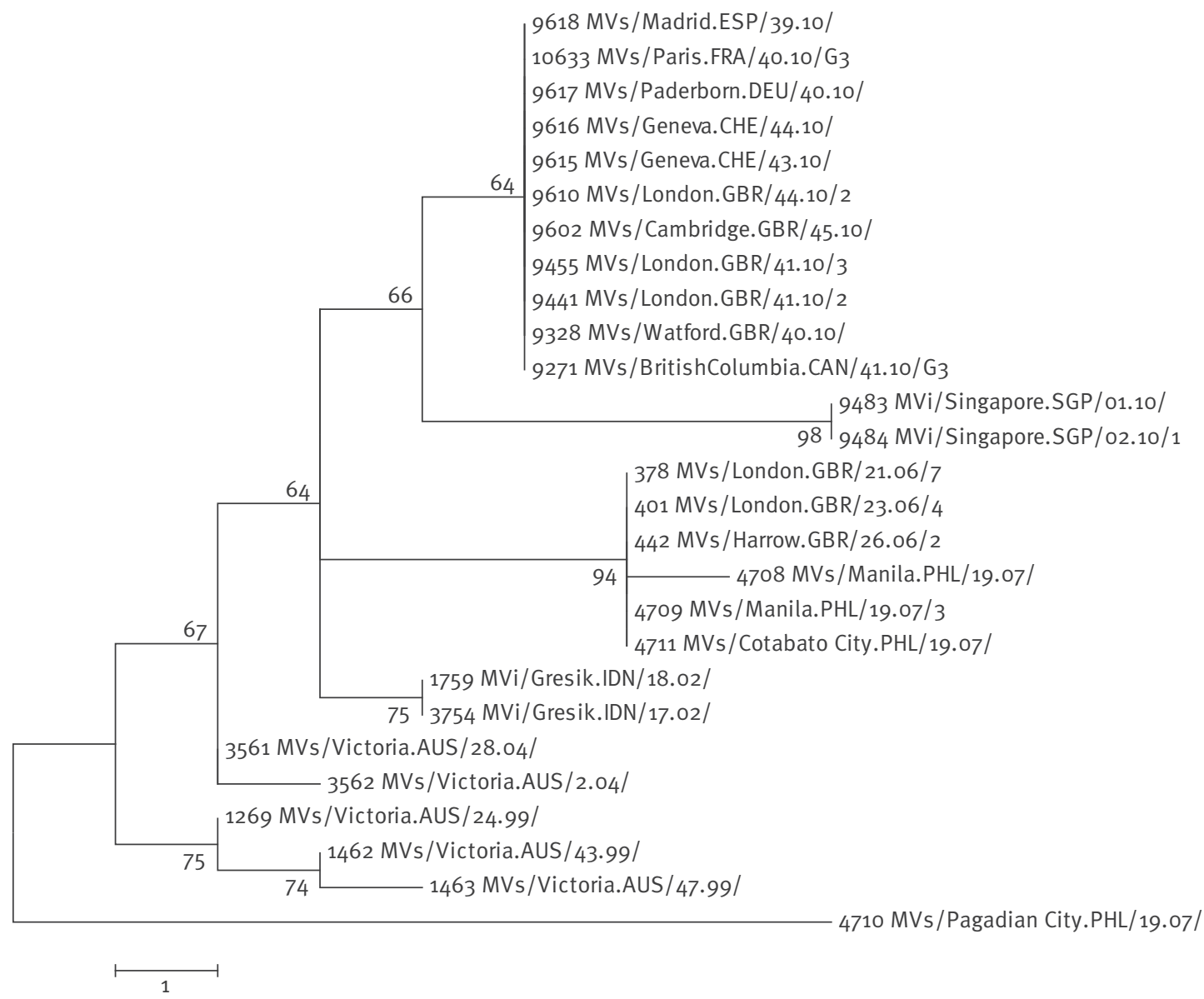

Numbers represent the ID number in the World Health Organization MeaNS database.

The tree was created using the number of nucleotide differences to generate a distance matrix and the neighbour-joining algorithm to cluster sequences, as implemented within MEGA5.

Branch lengths represent the number of nucleotide differences between each sequence and the resulting phylogeny was bootstrapped using 500 pseudo-replicates. 
to south-east Asia, were $100 \%$ identical over the N-450 nucleotide sequence window (Figure 2).

Interestingly, there were no other identical sequences in the WHO MeaNS database or GenBank. Two viruses with similar sequences (five nucleotides difference) had previously been identified in cases in Singapore in early 2010 and had links to Indonesia.

The cluster described in this paper highlights a number of important points. Not only is it essential to have an agreed standard nomenclature for describing viruses, both at genotype and at sequence level, but it is also critical that this information is shared. Within the WHO laboratory network of national, regional and global laboratories, genotype information is shared by regular reporting to the WHO Regional Offices of the sequencing results, and depositing sequence information in the WHO MeaNS database or GenBank. The MeaNS database is not only a repository for all reported measles sequences, but it also allows ready identification of identical or similar sequences in different countries, as in this case series. All laboratories performing measles genotyping and sequencing should be encouraged to submit their sequences in a timely manner to facilitate identification.

The cases described here also highlight how quickly measles viruses can spread, and become widely disseminated. Judging by the estimated dates of infection, it could be hypothesised that the person travelling on the plane with Patient $A$ was in the early stages of measles, and transmitted infection to the German copassenger and then to other cases in London, but that does not explain the index case in France. It is more likely that there were at least two independent importations of this $\mathrm{G}_{3}$ genotype to Europe (to France and the UK), and some early $\mathrm{G}_{3}$ infections have been missed. Almost certainly, the pocket of $\mathrm{G}_{3}$ measles infection in France has continued, with ongoing identification of $\mathrm{G}_{3}$ measles cases in France, as well as three further cases of $\mathrm{G}_{3}$ measles in the UK and two cases in Russia in February and March 2011, all with epidemiological links to infection in France. Similar small clusters of G3 measles cases have also recently been reported in Germany and the West Indies island of Saint Martin.

\section{Conclusions}

The number of identified $\mathrm{G}_{3}$ measles cases underestimates the true level of $\mathrm{G}_{3}$ measles activity in Europe, because not all infected individuals seek medical advice, and because in most countries samples suitable for genotyping are not routinely collected from all cases and therefore sequence information is not available. Routine collection of oral fluid from all cases with clinically suspected measles does allow confirmation of infection by serological or molecular methods and offers the potential for widespread genotyping to identify sources of importations and tracking of infection. Genotyping and molecular characterisation of circulating measles viruses are of increasing importance in confirming the absence of endemic infection in each country as Europe aims for the elimination of measles by $2015[1]$.

\section{Acknowledgments}

Thanks to all those who processed the samples, obtained the laboratory results and provided epidemiological information. Special thanks to: Antoaneta Bukasa (United Kingdom), Juan Emilio Echevarria, Juan Carlos Sanz and Luis Garcia Comas (Spain), Jean-Luc Richard and Pascal Cherpillod (Switzerland), Anette Siedler (Germany) and Rebecca Martin (World Health Organization Regional Office for Europe).

References

1. World Health Organization (WHO). Resolution. Renewed commitment to elimination of measles and rubella and prevention of congenital rubella syndrome by 2010 and Sustained support for polio-free status in the WHO European Region. Moscow, Russia, WHO Regional Office for Europe; 2010. Available from: http://www.euro.who.int/__data/assets/ pdf_file/0016/122236/RC60_eRes12.pdf

2. World Health Organization (WHO). Update of the nomenclature for describing the genetic characteristics of wild-type measles viruses: new genotypes and reference strains. Wkly Epidemiol Rec. 2003;27:229-39.

3. World Health Organization WHO). New genotype of measles virus and update on global distribution of measles genotypes. Wkly Epidemiol Rec. 2005;80(40):347-51.

4. World Health Organization (WHO). Global distribution of measles and rubella genotypes - update. Wkly Epidemiol Rec. 2006;81(51/52):474-9.

5. Rota PA, Brown KE, Mankertz A, Santibanez S, Shulga S, Muller CP, et al. Global Distribution of Measles Genotypes and Measles Molecular Epidemiology. J Infect Dis. Forthcoming 2011.

6. Gnaneshan S, Brown KE, Green J, Brown DW. On-line global/ WHO-European regional measles nucleotide surveillance. Euro Surveill. 2008;13(19):pii=18861. Available from: http://www. eurosurveillance.org/ViewArticle.aspx?Articleld=18861

7. World Health Organization (WHO). Monitoring progress towards measles elimination. Wkly Epidemiol Rec. 2010;85(49):490-5.

8. Mankertz A, Mulders M, Shulga S, Kremer J, Brown K, Santibanez S, et al. Molecular genotyping and epidemiology of measles virus transmission in the WHO European Region, 2007-10. J Infect Dis. Forthcoming 2011.

9. World Health Organization (WHO). Manual for the laboratory diagnosis of measles and rubella virus infection. 2 edition. Geneva:WHO. 2007. Available from: http://www.who.int/ immunization_monitoring/LabManualFinal.pdf

10. Institut de veille sanitaire (French Institute for Public Health Surveillance, INVS). Epidémie de rougeole en France. Données de declaration obligatoire en 2010 et données provisoires pour début 2011. [Measles outbreak in France. Mandatory notification data for 2010 and provisional data for the beginning of 2011]. INVS. 2011. [Accessed 28 Apr 2011]. French. Available from: http://www.invs.sante.fr/surveillance/ rougeole/Point_rougeole_220311.pdf 